\title{
Self reported symptoms and inhibition of acetylcholinesterase activity among Kenyan agricultural workers
}

Grace J A Ohayo-Mitoko, Hans Kromhout, James M Simwa, Jan S M Boleij, Dick Heederik

\begin{abstract}
Objectives-This study was part of the East African pesticides project. The general objective was to assess health hazards posed by handling, storage, and use of pesticides, on agricultural estates and small farms with a view to developing strategies for prevention and control of pesticide poisoning. The aim of this paper is to describe the prevalence of symptoms in this population, to relate levels of inhibition to reported symptoms and evaluate at which levels of inhibition symptoms become increased.

Methods-Complete data were available for 256 exposed subjects and 152 controls from four regions in Kenya. A structured questionnaire on symptoms experienced at the time of interview was given to all subjects and controls. Information was also obtained on sex, age, main occupation, and level of education. Symptoms reported during the high exposure period, were initially clustered in broader symptom categories from reference literature on health effects of pesticides that inhibit cholinesterase (organophosphate and carbamate). Prevalence ratios were estimated for symptoms with changes in cholinesterase activity in serum.
\end{abstract}

Results-Symptom prevalence in exposed subjects was higher during the high exposure period than the low exposure period, although these differences were not significant. Interestingly, a clear and significant change in symptoms prevalence was found in the controls with a higher prevalence in the low exposure period. Analysis of the relation between cholinesterase inhibition and symptoms showed that prevalence ratios were significantly $>1$ for respiratory, eye, and central nervous system symptoms for workers with $>30 \%$ inhibition. Similar results were found for analyses with the actual level of acetylcholinesterase activity.

Conclusion-The results suggest the presence of a relation between exposure and acetylcholinesterase inhibition, acetylcholinesterase activity, and respiratory, eye, and central nervous system symptoms. Increased symptom prevalence was found at acetylcholinesterase activities generally considered to be non-adverse.

(Occup Environ Med 2000;57:195-200)
Keywords: cholinesterase inhibition; symptoms; health effects; Kenya; agricultural workers

Cholinesterase monitoring is intended to prevent further exposure of asymptomatic workers with depressed cholinesterase activities, thereby preventing poisoning. ${ }^{1-3}$ Organophosphate and N-methyl carbamate pesticides inhibit cholinesterase, which results in an accumulation of acetylcholine in the nervous system. Decreases in colinesterase activity to $60 \%$ of baseline can produce relatively mild and non-specific symptoms such as vertigo, nausea, anxiety, vomiting, diarrhoea, asthmalike tightness of the chest, increased sweating, increased salivation, wheezing and shortness of breath, increased lacrimation, constriction of the pupils, and malaise. ${ }^{4-7}$ Severe poisoning resulting in greater depression of cholinesterase activity may result in unconsciousness, pulmonary oedema, respiratory failure, and death. ${ }^{4-12}$ Effects on the gastrointestinal system and bradycardia have also been associated with poisoning by pesticides that inhibit cholinesterase activity. ${ }^{13}$ Depressions resulting from organophosphate exposures can be cumulative, whereas depressions resulting from carbamate exposures are usually rapidly reversed. ${ }^{14} 15$

Although the effects of acute pesticide poisoning are well known, hardly any data exist on chronic effects, particularly neurotoxic and behaviourial aspects of organophosphate exposures. ${ }^{16-19}$ Clusters of neurological, rather than behaviourial diseases were the earliest sequelae noted after exposures to organophosphates. $^{20}$ Organophosphate induced delayed neuropathy has been found by Senanayake and Karaliedde. ${ }^{21}$ Respiratory paralysis followed the cholinergic phase of the illness in cases of poisoning with methamidophos and fenthion. Also weakness was noted in proximal limb muscles and muscles of the neck. Thompson and Stocks ${ }^{22}$ have shown the benefit of early otolaryngological consultation for the prevention and treatment of airway obstruction in patients with suspected organophosphate poisoning which can sometimes cause variant toxicity syndrome involving transient bilateral vocal cord paralysis.

The intermediate syndrome coincides with prolonged cholinesterase inhibition, and is not due to necrosis of the muscle fibres. When viewed together, the clinical and electromyographic features are best explained by combined presynaptic and postsynaptic dysfunc- 
tion of neuromuscular transmission. The intermediate syndrome is not related to an incipient delayed neuropathy. ${ }^{23}$

Many publications exist on exposure to organophosphates and carbamates and cholinesterase inhibition from several types of studies-such as case series, ${ }^{16}{ }^{24-26}$ individual case descriptions, ${ }^{27}$ and surveys. ${ }^{1}$ However, the relations between exposure, inhibition, and symptoms are not well established. The available evidence suggests that there is a high probability of adverse side effects below concentrations that are generally considered to be safe. ${ }^{42829}$

This study was part of the Kenyan component of a multicentre epidemiological survey, the East African pesticides project. ${ }^{30}$ The general objective was to assess the health hazards posed by handling, storage, and use of pesticides on agricultural estates and small farms in selected rural agricultural communities in Kenya where cotton, tobacco, flowers, and horticultural crops are grown, with a view to developing strategies for the prevention and control of pesticide poisoning.

Previously, we reported on acetylcholinesterase activity as an indicator of organophosphate and carbamate exposure in Kenyan agricultural workers. Among the exposed people, $29.6 \%$ had their cholinesterase activity depressed to values below $60 \%$ of baseline. ${ }^{31} \mathrm{~A}$ dramatic shift in acetylcholinesterase activity was apparent. The aim of this paper is to describe the prevalence of symptoms in this population, to relate levels of inhibition to reported symptoms and to evaluate at which levels of inhibition symptoms become increased.

\section{Materials and methods}

Selection of farms and estates was by multistage cluster sampling as described by OhayoMitoko et al. ${ }^{31}$ This study had a hybrid design that combined cross sectional and follow up design features. It was conducted in four regions of Kenya; Naivasha; Wundanyi; Homabay; and Migori. A preparatory phase was included to optimise the design for data collection.

The exposed group consisted of 623 agricultural workers, mainly applicators during the pesticide application periods, who were initially recruited into the study. The organophosphates used most often were dimethoate and malathion. Carbamates most often used were benomyi, mancozeb, methomyi, aldicarb, and propineb. Workers were followed up for at most 6 months, and the rate of follow up was $62.6 \%$ $(n=390)$. For 256 exposed subjects $(41.1 \%)$ complete data were available to study the relation between cholinesterase inhibition and self reported symptoms (health complaints), because questionnaires were not returned during either the first or second survey or because the questionnaires were poorly filled in. The control group consisted of 515 unexposed agricultural workers. These were sisal workers or subsistence farmers who were not occupationally exposed to pesticides. This group was as similar to the exposed group as possible for occupation, nutrition, and socioeconomic status. The rate of follow up was $53.6 \%(n=276)$. Complete data were available for 152 controls $(29.5 \%)$.

The study period was divided into periods of low and high exposure. During the low exposure period cholinesterase activity was measured in the subjects and controls to determine baselines for these people. Immediately after the work shift $10 \mathrm{ml}$ blood was taken by venepuncture, and was tested in the field with the method described later. All measurements of cholinesterase activity were performed by two state certified laboratory technicians (field assistants), blinded to exposure status. During the period of heavy pesticide application (high exposure), cholinesterase measurements were again performed on the subjects and controls by the same technicians. ${ }^{31}$ The acetylcholinesterase activity was measured with a World Health Organisation (WHO) approved field spectrophotometric kit, from EQM Research, based on the method of Ellman et al..$^{30}$ The variable used to describe change in acetylcholinesterase activity (expressed as \% inhibition) was derived as the ratio of acetylcholinesterase activity during high exposure divided by activity during low exposure. These variables are related to acetylcholinesterase activity determined in International Units (IU), $\mu \mathrm{mol}$ of substrate hydrolysed per $\mathrm{ml}$ blood at $25^{\circ} \mathrm{C}$ and corrected for haemoglobin concentration.

Written consent was obtained from the subjects and controls who agreed to participate, and they were allowed to drop out whenever they wanted. Study subjects identified as possibly having adverse health effects due to exposure to pesticides were informed of the situation and directed to go for medical treatment. The proposal was approved by the ethics review committee of the Kenya Medical Research Institute, Nairobi, Kenya.

A structured questionnaire in English was given by the investigators to all subjects and controls. The questions were sometimes translated orally into other languages by the interviewers as they were asked. The questionnaire consisted of both open and closed questions. Information was obtained on the sex of the worker, age, main occupation, and level of education. Additional questions were asked on symptoms experienced at the time of interview, with a checklist of 42 symptoms. Further questions were asked on whether or not some of these symptoms became more severe or frequent while working with pesticides or soon afterwards. Also, questions were asked on how long the last pesticide symptoms lasted and whether or not the respondents had symptoms that they thought might be due to pesticide poisoning and whether or not these occurred during or after the use of pesticides. Respondents were asked if they had ever had pesticide poisoning, and if so, the number of times that it had occurred and the details of the last episode, including the pesticide responsible for the poisoning episode by chemical group, chemical name, trade name, and the number of days the symptoms lasted. 
Table 1 General characteristics of the study population and change in concentration of acetylcholinesterase between low and high exposure (\% inhibition) and concentration of acetylcholinesterase during the high exposure period

\begin{tabular}{|c|c|c|c|c|c|c|c|}
\hline & \multicolumn{2}{|c|}{ Age (mean (SD)) } & \multicolumn{3}{|l|}{ Education (\%) } & \multicolumn{2}{|c|}{ Acetylcholinesterase (mean (SD)) } \\
\hline & Male & Female & No education & Elementary & Secondary & Inhibition (\%) & Activity (IU/ml) \\
\hline Exposed & $\begin{array}{l}34.0(13.0) \\
(\mathrm{n}=244)\end{array}$ & $\begin{array}{l}38.7(8.0) \\
(n=11)\end{array}$ & 0.4 & 63.9 & 29.7 & $0.65(0.12)$ & $4.17(0.82)$ \\
\hline Controls & $\begin{array}{l}37.2(13.6) \\
(\mathrm{n}=116)\end{array}$ & $\begin{array}{l}43.0(12.6) \\
(n=33)\end{array}$ & 16.9 & 59.6 & 23.5 & $0.96(0.08)$ & $6.02(0.64)$ \\
\hline
\end{tabular}

Symptoms reported during the high exposure period were clustered initially with reference to literature on health effects of pesticides (organophosphate and carbamate) that inhibit cholinesterase activity. This approach was used to support data reduction strategies.

Normal levels of acetylcholinesterase change (\% inhibition) was defined on the basis of the median inhibition in controls $(5.05 \%)$. Inhibition values below the median of controls were considered as normal (no inhibition $<5.05 \%$ ). Medium inhibition was defined as inhibition between $5.05 \%$ and $<30 \%$ (WHO cut off point). ${ }^{32}$ The high inhibition group was that with cholinesterase inhibition of $\geqslant 30 \%$.

Normal acetylcholinesterase activities during high exposure were also based on the median acetylcholinesterase activity in controls (>5.95 IU). A low acetylcholinesterase activity during the high exposure period was defined as a value $\leqslant 3.95 \mathrm{IU} / \mathrm{ml}$ for the exposed. Medium acetylcholinesterase activity level was defined as a value between 3.94 and $<5.95 \mathrm{IU} / \mathrm{ml}$.

All statistical analyses were done with SAS (version 6.11). Crude prevalences were compared with $\chi^{2}$ statistics. Changes over time were tested with the McNemar test to take into account the paired structure of the data. Associations between acetylcholinesterase inhibition and reported symptoms were evaluated by calculating prevalence rate ratios (PRRs) ${ }^{33}$ with Cox's proportional hazards model (PROC PHREG).

\section{Results}

Table 1 gives the general characteristics of the study population. Exposed workers were somewhat younger than controls and had received more education.

The main occupations of the subjects were farmer $(39.3 \%)$ and knapsack sprayer $(54.8 \%)$. Only $7.1 \%$ of the subjects reported that they had definitely had pesticide poisoning in the past whereas $40.1 \%$ reported having experienced symptoms due to pesticide poisoning, with only $25.4 \%$ seeking treatment. Of the subjects $9.1 \%$ reported that the symptoms occurred during pesticide use, $27.4 \%$ after use, and $7.9 \%$ reported having symptoms both during and after the use of pesticides. Of the subjects $22.2 \%$ reported that they knew of pesticide poisoning among the family and neighbours.

Of the subjects $91.7 \%$ indicated that the nature of their work with pesticides was as sprayers: $74.2 \%$ reported that they took some precautions when working with pesticides; $92.1 \%$ indicated that they washed or bathed immediately after the use of pesticides; $84.5 \%$ reported that they had a facility to wash or bathe; and $89.7 \%$ reported that they usually changed their clothes immediately after work. Although $46.4 \%$ of the subjects reported that they normally worked out the dose of pesticide to be used, more than half of the farmers and workers, especially from large scale farms, indicated that the foreman usually decided on the type of pesticide to be used. Of the farmers $71.4 \%$ reported that they stored pesticides and $87.3 \%$ of the subjects reported that their suppliers gave instructions on safe use of pesticides.

The following clusters were obtained for further analysis. The first group comprised symptoms of the skin and extremeties which included skin rash, itching, burning or prickling of the skin, tingling or numbness of hands, tingling and numbness of the face, muscular twitching or cramps in the face, muscular twitching and cramps around the neck, muscular twitching and cramps in the arms, and muscular twitching and cramps in the legs. Of the eight symptoms in the cluster, a positive score on three or more was used as a cut off point for further analysis. Respiratory symptoms included chest pain, cough, running nose, wheezing, difficulties in breathing, shortness of breath, and irritation of the throat (three or more out of seven were positive). Systemic symptoms included excessive sweating, nausea, vomiting, diarrhoea, excessive salivation, abdominal pain, burning on urination, and poor appetite (three or more out of eight were positive). Eye symptoms were lacrimation and irritation of the eyes (two out of two were positive). Symptoms of the central nervous system (CNS) included 14 items on difficulty in seeing, restlessness, difficulty in failing asleep, lacrimation, trembling of hands, and irritability (five or more out of 14 were positive).

The symptom prevalence was generally significantly higher during the high exposure period in exposed subjects than in controls, except for skin symptoms $\left(\chi^{2} p>0.10\right)$ and eye symptoms $\left(\left(\chi^{2} 0.05 \geqslant p<0.10\right.\right.$, table 2$)$. A clear and significant change in symptoms prevalence was found in the controls from the high to the low exposure period for respiratory symptoms and CNS symptoms (McNemar's test; $\mathrm{p}<0.05$ ), with a higher symptom prevalence during the low exposure period. For respiratory symptoms the difference between the two periods could be explained by a change in prevalence of shortness of breath, difficulties with breathing, wheezing, runny nose, and cough. The reduction in prevalence of CNS symptoms in the high exposure period could be explained by the change in prevalence of 
Table 2 Prevalence (\%) of clusters of symptoms during high and low exposure for both exposed $(n=259)$ and control subjects $(n=149)$

\begin{tabular}{|c|c|c|}
\hline Symptom & $\begin{array}{l}\text { High exposure } \\
\text { period }\end{array}$ & $\begin{array}{l}\text { Low exposure } \\
\text { period }\end{array}$ \\
\hline \multicolumn{3}{|c|}{ Skin or extremities $(\geqslant 3 / 8)$ : } \\
\hline Exposed & 12.4 & 14.3 \\
\hline Control & 10.1 & 11.4 \\
\hline \multicolumn{3}{|c|}{ Respiratory ( $\geqslant 3 / 7)$ : } \\
\hline Exposed & 19.3 & 14.7 \\
\hline Control & 5.4 & 14.8 \\
\hline \multicolumn{3}{|c|}{ Systemic $(\geqslant 3 / 8)$ : } \\
\hline Exposed & 15.1 & 12.4 \\
\hline Control & 8.1 & 11.4 \\
\hline \multicolumn{3}{|l|}{ Eye $(\geqslant 2 / 2)$ : } \\
\hline Exposed & 10.0 & 5.0 \\
\hline Control & 4.7 & 7.4 \\
\hline \multicolumn{3}{|c|}{ CNS $(\geqslant 5 / 14):$} \\
\hline Exposed & 19.7 & 17.4 \\
\hline Control & 8.7 & 17.4 \\
\hline
\end{tabular}

trembling hands, irritability, forgetfulness, restlessness, and difficulties failing asleep.

Of all 408 workers 167 had a high change in acetylcholinesterase inhibition $(\geqslant 30 \%)$ between the two exposure periods, 159 had an intermediate inhibition, 82 workers $(20.1 \%)$ had a normal acetylcholinesterase activity during high exposure, $127(31.1 \%)$ had an intermediate acetylcholinesterase activity, and $199(48.8 \%)$ had a low acetylcholinesterase activity. Most people with intermediate or high acetylcholinesterase inhibition, as opposed to intermediate or low acetylcholinesterase activity level, were exposed to cholinesterase inhibiting pesticides during the high exposure period.

Analysis of the relation between inhibition and symptoms showed that prevalence ratios (PRs) were significantly $>1$ for clustered respiratory and CNS symptoms for workers with $>30 \%$ change in inhibition (table 3 ), after correction for age and sex. Uncorrected PRs were generally lower. Prevalences for people with intermediate change in inhibition were increased for these clusters as well but they did not reach significance. Respiratory and CNS symptoms were also strongly related to acetylcholinesterase activity during the high exposure period. Also people with intermediate acetylcholinesterase activity during the high exposure period had a significantly increased

Table 3 Prevalence ratios (95\% CIs) for clusters of symptoms at day of interview by exposure group defined by change in acetylcholinesterase activity (\% inhibition) between the low and high exposure period adjusted for sex and age $(n=408)$

\begin{tabular}{llll}
\hline Symptom & $\begin{array}{l}\text { No inhibition } \\
(<5.05 \%)(n=8)\end{array}$ & $\begin{array}{l}\text { Medium inhibition } \\
(\geqslant 5.05 \%-<30 \%) \\
(n=75)\end{array}$ & $\begin{array}{l}\text { High inhibition } \\
(\geqslant 30 \%)(n=55)\end{array}$ \\
\hline Skin or extremities $(\geqslant 3 / 8)$ & 1 & $0.82(0.35$ to 1.94$)$ & $1.78(0.78$ to 4.08$)$ \\
Respiratory $(\geqslant 3 / 7)$ & 1 & $2.04(0.78$ to 5.38$)$ & $2.92(1.12$ to 7.61$)$ \\
Systemic $(\geqslant 3 / 8)$ & 1 & $1.20(0.50$ to 2.85$)$ & $1.94(0.82$ to 4.59$)$ \\
Eye $(\geqslant 2 / 2)$ & 1 & $0.89(0.27$ to 2.94$)$ & $2.92(0.97$ to 1.03$)$ \\
CNS $(\geqslant 5 / 14)$ & 1 & $2.56(0.99$ to 6.62$)$ & $3.29(1.26$ to 8.60$)$ \\
\hline
\end{tabular}

Table 4 Prevalence ratios (95\% CIs) for clusters of symptoms at day of interview by exposure group defined by acetylcholinesterase activity (IU/ml) during the high exposure period adjusted for sex and age $(n=408)$

\begin{tabular}{llll}
\hline Symptom & $\begin{array}{l}\text { Normal }(>5.95 \\
\text { IU/ml })(n=76)\end{array}$ & $\begin{array}{l}\text { Medium }(>3.95-\leqslant 5.95 \\
\text { IU/ml })(n=201)\end{array}$ & $\begin{array}{l}\text { Low }(\leqslant 3.95 \text { IU/mI }) \\
(n=131)\end{array}$ \\
\hline Skin or extremities $(\geqslant 3 / 8)$ & 1 & $1.77(0.71$ to 4.41$)$ & $1.98(0.76$ to 5.16$)$ \\
Respiratory $(\geqslant 3 / 7)$ & 1 & $4.10(1.24$ to 13.5$)$ & $4.17(1.23$ to 14.1$)$ \\
Systemic $(\geqslant 3 / 8)$ & 1 & $1.51(0.64$ to 3.55$)$ & $1.78(0.73$ to 4.35$)$ \\
Eye $(\geqslant 2 / 2)$ & 1 & $0.94(0.32$ to 2.72$)$ & $1.93(0.69$ to 5.42$)$ \\
CNS $(\geqslant 5 / 14)$ & 1 & $2.47(1.02$ to 5.95$)$ & $2.54(1.01$ to 6.36$)$ \\
\hline
\end{tabular}

prevalence of these symptom categories (table 4). Symptoms of the skin and extremities, and systemic and eye symptoms (low category only) were increased but the PR did not reach significance.

An internal analysis was applied to avoid the effect of systematic differences between exposed and control workers, by analysing relations between symptoms and acetylcholinesterase inhibition and activity within exposed people only. A significantly increased PRR was only found for eye symptoms with $\geqslant 30 \%$ change in inhibition and $\leqslant 5 \mathrm{IU} / \mathrm{ml}$ acetylcholinesterase activity. Interestingly, symptoms reported during the low exposure period were not related to acetylcholinesterase activity during the low exposure period.

\section{Discussion}

Results from this study show that many Kenyan agricultural workers have considerable changes in acetylcholinesterase inhibition and low acetylcholinesterase activities due to exposure to pesticides during the high exposure period. This study also shows that relations exist between change in cholinesterase inhibition and symptoms, especially respiratory symptoms, symptoms of the CNS (analysis including controls), and eye symptoms (internal analysis). The interpretation of these relations, however, is not unequivocal and is complicated by some methodological inadequacies in the study.

The change in prevalence of symptoms in controls from the high to the low exposure period is a key factor in explaining the relations between change in acetylcholinesterase activity and acetylcholinesterase activity during the high exposure period. The internal analysis (controls excluded) yielded a significant relation between change in acetylcholinesterase activity and acetylcholinesterase activity during the high exposure period for eye symptoms only. Only the analysis including controls showed strong relations between change in acetylcholinesterase activity and acetylcholinesterase activity during the high exposure period, and CNS and respiratory symptoms. However, in controls the symptom prevalence during the high exposure period was lower than in the low exposure period. This introduced a significant difference in symptom prevalence between exposed workers and controls during the high exposure period whereas the symptom prevalence did not differ between these two groups during the low exposure periods.

It could be argued that the increased prevalence for some symptoms in both exposed workers and controls during the low exposure period is caused by external non-occupational factors such as climate (temperature and humidity), and exposure to environmental dust. The low exposure period occurs during the hot and dry season of the year. During this period fields and roads are dry and dust is suspended in the air by the wind. The high dust concentrations might explain some of the respiratory symptoms. The high prevalence of symptoms especially for irritability, forgetfulness, restlessness, and difficulties failing asleep, 
could be explained by the high temperature during this period. Symptoms in controls due to the high temperatures and environmental dust disappear during the high exposure period but remain in exposed workers because of exposure to organophosphates and carbamates. This explanation is also supported by the observation that \% inhibition as well as acetylcholinesterase activity are normal in controls during the high and low exposure period but change considerably over time in exposed workers. $^{31}$

The present study also suggests that an increased symptom prevalence might occur at acetylcholinesterase activities that are generally considered to be not adverse. However, because of the methodological problems already mentioned this finding needs support by additional evidence from another independent study that included objective health variables such a spirometry and nerve conduction.

We evaluated the potential influence of different forms of bias. Selection bias cannot be ruled out and might have biased the outcomes of this study to some extent. However, the sample used for this study $(n=408)$ has a similar age distribution to those eligible for follow up $(n=666)$ and the source population $(n=1138) .{ }^{31}$ Most of the data were self reported, which could introduce a strong information (responder) bias, because the facts might have been distorted by the people involved, including employers, either in the hope of secondary gain or to avoid adverse outcomes - such as being fired or investigated by the authorities. Some of the subjects consisted of groups of workers from large farms and their input could reflect a psychogenic group consensus rather than the individual person's unbiased assessment. Although independent information on the validity of the questionnaire items is not available, it seems unlikely that responder bias can explain the differences in symptom prevalence between exposed and control workers and the relations between acetylcholinesterase inhibition and acetylcholinesterase activity during high exposure and symptoms. The relations between symptoms and the potential confounders age and sex had the expected directions (increased prevalence for women and increasing prevalence with increasing age) and these relations would also have been distorted if considerable responder bias were present. It is also possible that some of the relations might have been confounded by the effects of smoking. Information about smoking habits was not available for both controls and exposed workers and correction for differences in smoking habits between exposed workers and controls was therefore not possible. This could especially have affected the relations found for respiratory symptoms. However, it is unlikely that differences in smoking habits can account for the large differences in prevalence of respiratory symptoms between controls and exposed workers during the high exposure period. Controls came from the same area as the exposed workers. The age distributions were similar as was the educational level. This makes the existence of large differences in smoking habits unlikely.

Although most of the exposed subjects $(91.7 \%)$ indicated that their work involved pesticides, only $10 \%$ reported experiencing these symptoms during spraying. About one quarter reported experiencing symptoms after use. This may have reduced the number of symptoms reported. However, about $22.2 \%$ reported that they knew of pesticide poisoning among the family and neighbours, indicating that this is a serious problem in the community and requires immediate attention.

In conclusion, results of this study suggest the presence of a relation between the change in acetylcholinesterase inhibition from low to high exposure periods, and acetylcholinesterase activity during high exposure and respiratory, eye, and CNS symptoms. Increased symptom prevalence was found at acetylcholinesterase activities which are generally considered to be not adverse. This suggests the need for more health protective threshold levels that are more sensitive than the recommended WHO threshold of $70 \%$ of baseline red blood cell cholinesterase activity of baseline activity (30\% inhibition), and which are applicable at the population level. However, this relation requires confirmation in an independent study with more objective health variables because of changes in symptom prevalence in unexposed controls.

1 Brown SK, Ames RG, Mengle DC. Occupational illnesses from cholinesterase inhibiting pesticides among agricultural applicators in California, 1982-5. Arch Environ Health $1989 ; 44: 34-9$

2 Lopez-Carillo L, Lopez-Cervantes M. Effect of exposure to organophosphate pesticides on serum cholinesterase levels. Arch Environl Health 1993;48:359-63.

3 Lessenger JE. The pesticide-exposed worker: an approach to the office evaluation. F Am Board Fam Practice 1993;6:3341.

4 Ames RG, Brown SK, Mengle DC, et al. Cholinesterase activity depression among California agricultural pesticide activity depression among California agriculturs. Am f Ind Med 1989;15:143-50.

5 Markowitz SB. Poisoning of an urban family due to misapplication of household organophosphate and carbamate plication of household organophosphate and carba
pesticides. F Toxicol Clin Toxicol 1992;30:295-303.

pesticides. F Toxicol Clin Toxicol 1992;30:295-303.
6 Padilla S. Regulatory research issues related to cholinestPadilla S. Regulatory research issues related to
erase inhibition. Toxicology 1995;102:215-20.

erase inhibition. Toxicology 1995;102:215-20.
7 Lotti M. Cholinesterase inhibition: complexities in interpretation. Clin Chem 1995;41:1814-18.

8 Namba T. Cholinesterase inhibition by organophosphorus compounds and its clinical effects. Bull World Health Organ 1971;44:289-307.

9 Khan AS, Ali SA. Assessment of certain hematological responses of factory workers exposed to pesticides. Bull Environ Contam Toxicol 1993;51:740-7.

10 Hayes WJ. Pesticides studied in man. Baltimore: Williams and Wilkins, 1982

11 Kaloyanova F. Interactions of pesticides. In: Health effects of combined exposures to chemicals in work and community environments. Copenhagen: WHO, Regional Office for Europe, 1983:165-95. (Interim document 11.)

12 Coye MJ, Barnett PG, Midtling JE, et al. Clinical confirmation of organophosphate poisoning by serial cholinesterase tion of organophosphate poisoning by serial
analysis. Arch Intern Med 1987;47:438-42.

13 Kashyap SK, Jani JP, Sayyed HN, et al. Clinical effects and cholinesterase activity changes in workers exposed to phorate (thimet). F Environ Sci Health B 1984;19:479-89.

14 Gage JC. The significance of blood cholinesterase activity measurements. Residue Review 1967;18:159.

5 Vandekar M, Plestina R, Wilhern K. Toxicity of carbamates for mammals. Bull World Health Organ 1971;44:241-9.

16 Davies E J. Neurotoxic concerns of human pesticide exposures. Am f Ind Med 1990;18:327-31.

17 Forget G. Pesticides and the third world. $\mathcal{f}$ Toxicol Environl Health 1991;32:11-31.

18 Robinson DG, Trites DG, Banister EW. Physiological effects of work stress and pesticide exposure in tree planting by British Columbia silviculture workers. Ergonomics 1993;36:951-61.

19 Kishi M, Hirschhorn N, Djajadisastra M, et al. Relationship of pesticide spraying to signs and symptoms in Indonesian farmers. Scand $\mathcal{f}$ Work Environ Health 1995;21:124-33. 
20 Xintaras C, Burg JR, Tanaka S, et al. Occupational exposure to leptophos and other chemicals. Cincinnati, OH: DHEW 1978:78-136.

21 Senanayake N, Karaliedde L. Neurotoxic effects of organophosphorus insecticides. N Engl F Med 1987;316:761-3.

22 Thompson JW, Stocks RM. Brief bilateral vocal cord paralysis after insecticide poisoning. A new variant of toxicity syndrome. Arch Otolaryngol Head Neck Surg 1997;123:936.

23 De Bleecker J, Van den Neucker K, Colardyne F. Intermediate syndrome in organophosphorus poisoning: a prospective study. Crit Care Med 1993;11:1706-11.

24 Lander F, Lings S. Variation in plasma cholinesterase activity among greenhouse workers, fruitgrowers, and slaughtermen. Br F Ind Med 1991;48:164-6.

25 Maroni M, Jarvisalo J, la Ferla F. The WHO-UNDP epidemiological study on the health effects of exposure to orga-
nophosphorus pesticides. Toxicol Lett 1986;33:115-23.

26 Coye MJ, Lowe JA, Maddy KT. Biological monitoring of agricultural workers exposed to pesticides: I. Cholinestagricultural workers exposed to pesticides: I. Cholinest27 .

27 Lessenger JE, Estock MID, Younglove T. An analysis of 190 cases of suspected pesticide illness. F Am Board Fam Practice 1995;8:278-82.
28 Ames RG, Brown SK, Mengle DC, et al. Protecting agricultural applicators from over-exposure to cholinesterasenhibiting pesticides: perspectives from the California programme. f Soc Occup Med 1989;39:85-92.

29 Popendorf JW. Effects of organophosphate insecticide esidue variability on re-entry intervals. Am f Ind Med 1990;18:313-9.

30 Ohayo-Mitoko GJA, Simwa JM, Omondi BEO, et al. The East African Pesticides Network (Kenya); A Preliminary report on "Pattems of pesticide use and assessment of health hazards on "Pattems of pesticide use and assessment of health hazards
caused by pesticide handling, storage and use on agricultural caused by pesticide handing, storage and use on agricultural estates and small farms in selected communities in

31 Ohayo-Mitoko GJA, Heederik DJJ, Kromhout H, et al. Acetylcholinesterase inhibition as an indicator of organophosphorus and carbarnate poisoning in Kenyan agricultural workers. International fournal of Environmental and Occupational Health 1997;3:210-20.

32 World Health Organisation. WHO Permanent Commission and International Association on Occupational Health, Sub-Committee on Pesticides: epidemiological toxicology of Sub-Committee on Pesticides: epidemiological toxicology of
pesticide exposure. Arch Environ Health 1972;25:399-405.

33 Lee J, Chia KS. Estimation of prevalence rate ratios for cross sectional data. An example in occupational epidemiology. Br f Ind Med 1993;50:861-4 